\title{
Educación disruptiva: Nuevos desafíos en la formación de investigadores sociales en tiempos de pandemia y distanciamiento social
}

\section{Disruptive education: New challenges in the training of social researchers in pandemic and social distancing contexts}

\author{
David Ocampo-Eyzaguirre, ${ }^{1}$ Wilson Sucari, ${ }^{2 *}$ Julissa Anaya, ${ }^{3}$ Roxana Medina, ${ }^{4}$ \\ Higinio Zuñiga-Sánchez ${ }^{5}$ \\ Universidad Autónoma Tomás Frías, Potosí, Bolivia ${ }^{1}$ \\ Universidad Nacional de Huancavelica, Huancavelica, Perú ${ }^{2}$ \\ Pontificia Universidad Católica del, Lima, Perú ${ }^{3}$ \\ Universidad Nacional del Altiplano, Puno, Perú ${ }^{4}$ \\ Universidad Nacional del Altiplano, Puno, Perú ${ }^{5}$ \\ iD ORCID ID: https://orcid.org/0000-0003-2406-7544 ${ }^{1}$ \\ (iD) ORCID ID: https://orcid.org/0000-0001-5874-0966 $^{2}$ \\ ORCID ID: https://orcid.org/0000-0003-2424-984X ${ }^{3}$ \\ ORCID ID: https://orcid.org/0000-0003-2237-11984 \\ ORCID ID: https://orcid.org/0000-0002-8204-1847
}

Recibido: 08 de junio de 2021

Aceptado:02 de setiembre de 2021

\begin{abstract}
Resumen
Sin duda, la emergencia sanitaria y el distanciamiento social han afectado de manera directa los procesos de formación de investigadores, así como la aplicación de estrategias investigativas, principalmente en el campo de las ciencias sociales, donde la relación y la interacción en los procesos investigativos son un aspecto importante. En este contexto, el objetivo del estudio fue determinar posibles estrategias investigativas que permitan contribuir a fortalecer procesos de investigación científica en tiempos de pandemia y distanciamiento social en las ciencias sociales. El trabajo se basa en el paradigma naturalista interpretativo, con un enfoque de investigación cualitativa. Entre los principales hallazgos se destaca la diversidad de definiciones respecto a la educación disruptiva. En relación con la formación de investigadores se evidencian que se utilizan estrategias individuales y grupales que permiten fortalecer sus competencias investigativas, en relación con las estrategias investigativas se han generado y adecuado nuevas
\end{abstract}


estrategias. Se concluye que, a partir de la educación disruptiva, existen nuevos desafíos en la formación de investigadores y el manejo de nuevas estrategias de indagación, en tiempos de pandemia y distanciamiento social.

Palabras clave: Educación disruptiva, estrategias de investigación, distanciamiento social, formación de investigadores.

\begin{abstract}
Undoubtedly, the health emergency and social distancing has directly affected the training processes of researchers, as well as the application of research strategies, mainly in the field of social sciences, where the relationship and interaction in research processes are an important aspect. In this context, the objective of this study was to determine possible investigative strategies that allow to contribute strengthening scientific research processes in times of pandemic and social distancing in the Social Sciences. The work is based on the interpretive naturalistic paradigm, with a qualitative research approach. Among the main findings, the diversity of definitions stands out regarding disruptive education, in relation to the training of researchers, it is evident that individual and group strategies are used that allow strengthening their investigative competences, in relation to investigative strategies they have generated and adapted new strategies because of the COVID-19 pandemic. From disruptive education, it is concluded that there are new challenges in the training of researchers and the management of new investigation strategies, in times of pandemic and social distancing.
\end{abstract}

Keywords: Disruptive education, research strategies, social distancing, researcher training.

\title{
Introducción
}

Las formas de encarar los procesos de investigación social a partir de la emergencia sanitaria y el distanciamiento social han producido diversos cambios y modificaciones, estableciendo nuevas formas de relacionamiento e interacción social (Bonilla-Guachamín, 2020; Millán \& Núñez-Cortés, 2020). Es así como los postulados de la educación disruptiva se ponen en evidencia, estableciendo nuevos desafíos en la formación de investigadores sociales en tiempos de pandemia y distanciamiento social, donde es muy difícil establecer una relación directa con los involucrados de la investigación, sean estas comunidades, grupos sociales, familias o individuos.

En este aspecto, la educación superior enfrenta grandes desafíos a partir de la emergencia sanitaria y el distanciamiento social debido a la pandemia de la COVID 19 (Alvarez et al., 2021; Elgueta, 2020; García-Morales et al., 2021). Si se considera que una disrupción está relacionada a una rotura o una interrupción brusca, la educación disruptiva es aquella que pretende romper 
con lo establecido o las estructuras tradicionales para mejorar los procesos educativos en función de las características del actual contexto, basados en las nuevas tecnologías de información y de comunicación (Abdel-Basset et al., 2021; Molano, 2018; Piyatamrong et al., 2021).

En base a lo mencionado, la educación disruptiva consiste en una nueva forma de realizar los procesos de aprendizaje-enseñanza a partir del uso de estrategias diferentes a las tradicionales, en muchos casos estas están acompañadas por las nuevas tecnologías de información y de comunicación (NTIC). La importancia y la necesidad del uso de estas tecnologías se hicieron más evidentes y necesarias en esta etapa de la pandemia de la COVID 19 y el distanciamiento social que surgió a partir de la emergencia sanitaria.

La importancia del uso de la tecnología en la actualidad es innegable, que se manifiesta en un cambio intenso, tanto en los procesos de aprendizaje y enseñanza en el contexto de la educación superior como en la formación de investigadores (enseñanza de la investigación científica) en el campo de las ciencias sociales. A esto se suma los retos y desafíos que suponen las nuevas tecnologías facilitadores de los procesos de enseñanza, las universidades son las formadoras y proveedoras de profesionales, que en el mundo actual, deben tener sensibilidad social y estar preparados intelectualmente para asumir desafíos y tener capacidad de resolver problemas (Camacho, 2010).

Estos aspectos hacen necesaria una profunda reflexión de la educación superior en la actual coyuntura, fundamentalmente, en la enseñanza y la formación de investigadores en el campo de las ciencias sociales. A partir de ello, se plantea a la educación disruptiva como una alternativa que permita desarrollar las competencias y las habilidades investigativas. La educación disruptiva se basa en los principios de la educación personalizada, en la individualidad en los procesos de enseñanza de la investigación científica y la formación de investigadores; en el uso de herramientas digitales, de las TIC y los medios informáticos como un medio para transmitir conocimientos y desarrollar determinadas competencias y habilidades en los estudiantes (Cabrol \& Severin, 2010; García-Morales et al., 2021; Molano, 2018).

Por último, el aprendizaje a partir de la experiencia, uno de los principales cambios que busca la educación disruptiva, es la enseñanza a partir de la práctica; buscando que el estudiante realice el aprendizaje a través de la experiencia (Molano, 2018). En la formación de investigadores sociales, este es un aspecto muy importante, por lo que el estudiante debe aprender la investigación a partir de la capacitación y el entrenamiento. 
Lo anterior pone en evidencia que como menciona De Giusti (2021), la formación de investigadores en un determinado país, ha estado y está influenciado por dos aspectos: el papel fundamental de las universidades y el rol del Estado; a partir del fortalecimiento de los institutos de investigación en función de fortalecer los procesos investigativos en tecnología y en determinadas áreas; sin embargo, estos procesos de investigación social y las formación de investigadores sociales en el nivel de grado (Licenciatura) se han visto limitados $\mathrm{y}$ obstaculizados por la pandemia del COVID - 19 y el distanciamiento social.

En este caso, la formación en investigación dentro de las ciencias sociales constituye un elemento muy importante del proceso de formación de los nuevos profesionales. Esta labor está orientada a la asimilación de un conjunto de operaciones y procedimientos metodológicos de la investigación científica, así como la recolección de la información de campo, el procesamiento y el análisis de los resultados de la investigación (Landazábal et al., 2013). Desarrollar procesos de aprendizaje-enseñanza de la investigación científica y la formación de investigadores en ciencias sociales en épocas de pandemia y el distanciamiento social producto del COVID - 19, no es tarea fácil. Asumir estos procesos en tiempos de crisis sanitaria, donde se limita fundamentalmente el trabajo de campo, recolección de la información y la interacción entre el investigador y los sujetos de investigación (comunidades, grupos, familias e individuos) trae consigo una serie de inquietudes en el docente y el estudiante (Ordorika, 2020). La formación de investigadores en el mundo contemporáneo ha estado condicionado históricamente por dos variables: las tradiciones de la Educación Universitaria y el tipo de política de la ciencia, la investigación y el desarrollo impulsada por el gobierno (Fernández, 2002; Rivas, 2004).

Por otro lado, Gonzales et al. (2004) observa que las universidades tienen como función principal realizar investigación, la que provee al estudiante un conocimiento de la realidad y favorece el proceso de aprendizaje; asimismo ocurre la vinculación del futuro profesional a la sociedad de forma orgánica. Por ello, a partir de las consideraciones anteriores, se hace evidente la importancia que asume el proceso aprendizaje enseñanza de la investigación científica, como un proceso de formación de investigadores en el contexto de la educación superior. En este proceso la labor docente es muy importante, tal como manifiesta Hernández et al. (2014), los docentes deben acompañar al estudiante en su proceso de investigación, demostrando creatividad en su metodología y permitiéndose aprender conjuntamente con sus alumnos. 
A partir de estas consideraciones, las estrategias de enseñanza de la investigación se conciben como procedimientos didácticos utilizados por el docente, a fin de producir en los estudiantes determinadas experiencias, secuencia de actividades del docente y acciones alternativas del docente para responder de manera adecuada a determinadas expresiones de los estudiantes (Vélez \& Pérez, 2019). Es así como las estrategias de aprendizaje y enseñanza son un conjunto de procedimiento que se relacionan con las actividades de transmisión de conocimientos y experiencias del docente hacia el estudiante.

Conforme al objeto de estudio de la presente investigación, se refiere a las estrategias de investigación social como un proceso por el cual se llega al conocimiento en el campo de la realidad social; es decir, de algún problema social concreto. De acuerdo a Ander-Egg (2011), la metodología tiene implicaciones de carácter ontológico, epistemológicas y de carácter lógico. En su carácter ontológico se hace referencia a la naturaleza del objeto social, es decir, al tipo y a la clase de realidad que se aborda. Las implicaciones de carácter epistemológico se refieren a las posibilidades del conocimiento, su modo de producción y sus formas de validación, que establecen o determinan la relación entre la realidad y el pensamiento (relación sujeta - objeto) (Ander-Egg, 2011). Por último, las implicaciones de carácter lógico se refieren a los métodos y procedimientos que utiliza el ser humano para llegar a un conocimiento.

En base a las consideraciones planteadas, es muy importante plantear y formular estrategias de investigación para estos tiempos de crisis sanitaria y pandemia del COVID-19, que permita desarrollar estudios en las ciencias sociales que permitan desarrollar procesos de recolección de información, a partir de la aplicación de un conjunto de estrategias investigativas, cumpliendo con el cuidado de la salud de los investigadores sociales, y los aspectos procedimentales y metodológicos de la investigación científica. A la luz de este contexto general, el objetivo del presente estudio es determinar posibles estrategias investigativas que permitan contribuir a fortalecer procesos de investigación científica en tiempos de pandemia y distanciamiento social en el área de las ciencias sociales.

\section{Métodos y materiales}

Este trabajo se ha desarrollado bajo el paradigma naturalista interpretativo, enmarcado en el enfoque cualitativo y de tipo fenomenológico (Dibley et al., 2018; Ho et al., 2017; Iivari, 2018). La técnica utilizada para la recolección de la información fue la entrevista a profundidad 
a informantes claves (Beaud, 2018; González-Revaldería et al., 2017; Sordini, 2018), especialistas en educación superior, durante los meses de julio y agosto de 2020. Debido a la pandemia COVID 19, las entrevistas a profundidad se desarrollaron mediante Zoom y Google Meet, con el añadido de preguntas complementarías por Google Forms. Estas se aplicaron a 10 expertos de cuatro universidades bolivianas: Universidad Autónoma "Tomás Frías (Potosí), Universidad Mayor de San Simón (Cochabamba), Universidad Central de CochabambaUNICEN (Cochabamba) y de la Universidad Pública del Alto UPEA (El Alto - La Paz).

Los especialistas (informantes claves) fueron seleccionados tomando en cuenta los siguientes criterios: experiencia en el área de educación superior, especialistas en educación superior con grado académico de magíster o doctor. En este caso, el análisis profundo de las entrevistas permitió establecer tres categorías de análisis: educación disruptiva, formación de investigadores y estrategias de investigación (ver tabla).

\section{Tabla 1}

Ejes y unidades de análisis

\section{Universo de investigación Categorías de análisis Unidades de estudio}

Educación disruptiva:
Nuevos desafíos en la
formación de investigadores
sociales en tiempos de
pandemia y distanciamiento
social.

Formación investigadores

Estrategias investigación
- Aprendizaje personalizado.

- Aprendizaje basado en medios tecnológicos.

- Aprendizaje en las necesidades de individuo.

- Formación de redes de investigadores comunidades de práctica.

de - Proceso de formación de investigadores

- Estrategias de investigación individual

- Estrategias de investigación grupal.

de - Investigación cuantitativa

- Investigación cualitativa 


\section{Consideraciones éticas}

Los entrevistados participaron con previo consentimiento informado. Aceptaron participar voluntariamente en las entrevistas virtuales.

\section{Resultados}

Se procedió analizar y sistematizar la información, tanto a nivel descriptivo y cualitativo, lo que permitió desarrollar comparaciones e interpretaciones. La sistematización y procesamiento de resultados se realizó utilizando el software de procesamiento de datos cualitativos ATLAS. ti (v. 7).

En concordancia con los ejes de análisis, a continuación se presentan los resultados de las entrevistas a profundidad desarrolladas en el proceso de la investigación. Los resultados evidencian una descripción de la educación disruptiva, sus principios, el proceso de la formación de investigadores y las estrategias investigativas posibles a ser utilizadas en las investigaciones sociales en tiempos de pandemia y distanciamiento social; a partir, de la educación disruptiva.

\section{Educación disruptiva}

De acuerdo con las entrevistas de profundidad realizadas a especialistas y/o expertos en investigación científica y educación respecto a la educación disruptiva, se pudo evidenciar que los especialistas la entienden como una forma de educación que se relaciona a la interrupción de la enseñanza tradicional:

[...] La educación disruptiva se trata de un proceso educativo, en la cual existe una interrupción de la educación tradicional; es decir, es una nueva propuesta educativa, en procura de construir una educación diferente en función a las exigencias del contexto. Es una nueva forma de entender y realizar la práctica educativa; a partir de la utilización de las nuevas tecnologías de información y de comunicación. Se trata de una nueva concepción de la educación a partir de una interrupción de la educación tradicional [...] cuyo propósito es de fortalecer la educación, desde nuevas prácticas, en función de las demandas y las exigencias de la sociedad (Docente de Posgrado Universidad Pública del Alto UPEA)

Del mismo modo, se percibe que las actividades académicas deben tener un carácter más personalizado; es así como en el proceso de la investigación y producto de las entrevistas a especialistas en relación con el tema de investigación, se evidencia que la formación de investigadores sociales en tiempos de pandemia y distanciamiento social requiere de un proceso personalizado; a partir de, la utilización de recursos tecnológicos y redes sociales. 


\section{Principios de la educación disruptiva}

Con la finalidad de asumir una nueva forma de educación, basados en los principios de una educación personalizada, aprendizaje mediado por la TIC y el aprendizaje basado en la experiencia y las prácticas. Los especialistas y expertos en relación con el tema de investigación coincidieron que la educación disruptiva se basa en un conjunto de principios:

Una forma de aprendizaje diferente a la educación tradicional, como es la educación disruptiva se basa en una serie de orientaciones que permiten desarrollar un aprendizaje significativo [...] está basado en una educación personalizada, basada en la tecnología y la formación de comunidades de práctica, centrados en la colaboración de los estudiantes. (Entrevista a experto - Universidad Mayor de San Simón - Cochabamba).

Los principios expuestos por los expertos y especialistas son concordantes con los postulados de Abdel-Basset et al. (2021), Molano (2018), entre otros, quienes plantean que ,efectivamente, la educación disruptiva es una nueva forma de encarar procesos de aprendizaje y enseñanza, en función a un conjunto de directrices, características y principios que la convierten en una nueva forma de entender a la educación en diferentes entornos.

\section{Tabla 2}

Principios de la Educación Disruptiva

\begin{tabular}{|c|c|}
\hline Principios & Características \\
\hline Aprendizaje personalizado & $\begin{array}{ll}\text { - } & \text { Atención a la diversidad } \\
\text { - } & \text { Orientación personal } \\
\text { - } & \text { Educación en valores }\end{array}$ \\
\hline $\begin{array}{l}\text { Aprendizaje basado en medios } \\
\text { tecnológicos }\end{array}$ & $\begin{array}{l}\text { - } \quad \text { Rompe los límites de la clase tradicional } \\
\text { - Convierte a los estudiantes en creadores y } \\
\text { consumidores de la información. } \\
\text { - } \\
\text { Desarrolla actividades colaborativas. }\end{array}$ \\
\hline $\begin{array}{l}\text { Aprendizaje en las necesidades de } \\
\text { individuo. }\end{array}$ & $\begin{array}{l}\text { - Aprendizaje individualizado } \\
\text { - Identificación de fortalezas y debilidades de } \\
\text { los estudiantes. } \\
\text { - Atención personalizada. }\end{array}$ \\
\hline $\begin{array}{l}\text { Formación de redes de investigadores } \\
\text { y comunidades de práctica }\end{array}$ & $\begin{array}{l}\text { - } \text { Trabajo en equipo } \\
\text { - Motivación a la formación de redes de } \\
\text { investigadores online. } \\
\text { - Comunidades colaborativas. }\end{array}$ \\
\hline
\end{tabular}

Fuente: Elaboración propia 
Los elementos señalados en la tabla 2, en concordancia con datos recogidos a partir de las entrevistas realizadas a expertos, permiten determinar un conjunto de principios que orientan los procesos de la educación disruptiva. Todo estos contribuyen a desarrollar un proceso educativo en el actual contexto de distanciamiento social, basado en una educación personalizada, en la utilización de medios tecnológicos y las nuevas plataformas de enseñanza aprendizaje. Además, se sostiene sobre las necesidades propias de los estudiantes, apoyando a la formación de redes de investigación (trabajos en comunidades y redes), utilizando los medios tecnológicos de una manera proactiva.

\section{Formación de investigadores}

Respecto a la categoría de formación de investigadores en el área de las ciencias sociales en épocas de pandemia y distanciamiento social, las entrevistas a profundidad realizadas a expertos dan cuenta que, los procesos de formación de investigadores nóveles (estudiantes de investigación científica) es un pilar fundamental en el proceso de la educación superior.

La formación de investigadores sociales en tiempos de pandemia y distanciamiento social requiere de un compromiso de los docentes [...] requiere, además, de la utilización de los medios tecnológicos que faciliten el proceso de fortalecimiento de habilidades y competencias en la formación de investigadores nóveles en el área de las Ciencias Sociales. [...] la formación de investigadores nóveles es un pilar fundamental de la Educación Superior (Experto, Universidad Autónoma “Tomás Frías”).

De acuerdo con la opinión de los expertos/especialistas, la influencia de la formación de investigadores se da partir de dos escenarios: las universidades y las políticas de estado. Las universidades cumpliendo su rol fundamental de investigación, desarrollan procesos de formación de investigadores en diferentes niveles (grado - posgrado); por otro lado, el estado cumple un rol importante en la formación de investigadores, a partir de un conjunto de políticas de estado expresados en programas de investigación; sin embargo esta situación se aplican de distinta manera de un país a otro, en el caso particular de Bolivia, en este aspectos existe algunas deficiencias, tomando en cuenta que existen pocas políticas y programas del estado que contribuyan a la formación de investigadores, si existen acciones dirigidas a la formación de investigadores en los distintos niveles es a partir de las Universidades. Por otro lado, el proceso de formación de investigadores se desarrolla a partir de un conjunto de estrategias, las mismas 
que se expresan a nivel individual y a nivel de grupo, tal y como se ha podido evidenciar en el proceso de la presente investigación (Figura 1)

Figura 1

Formación de investigadores

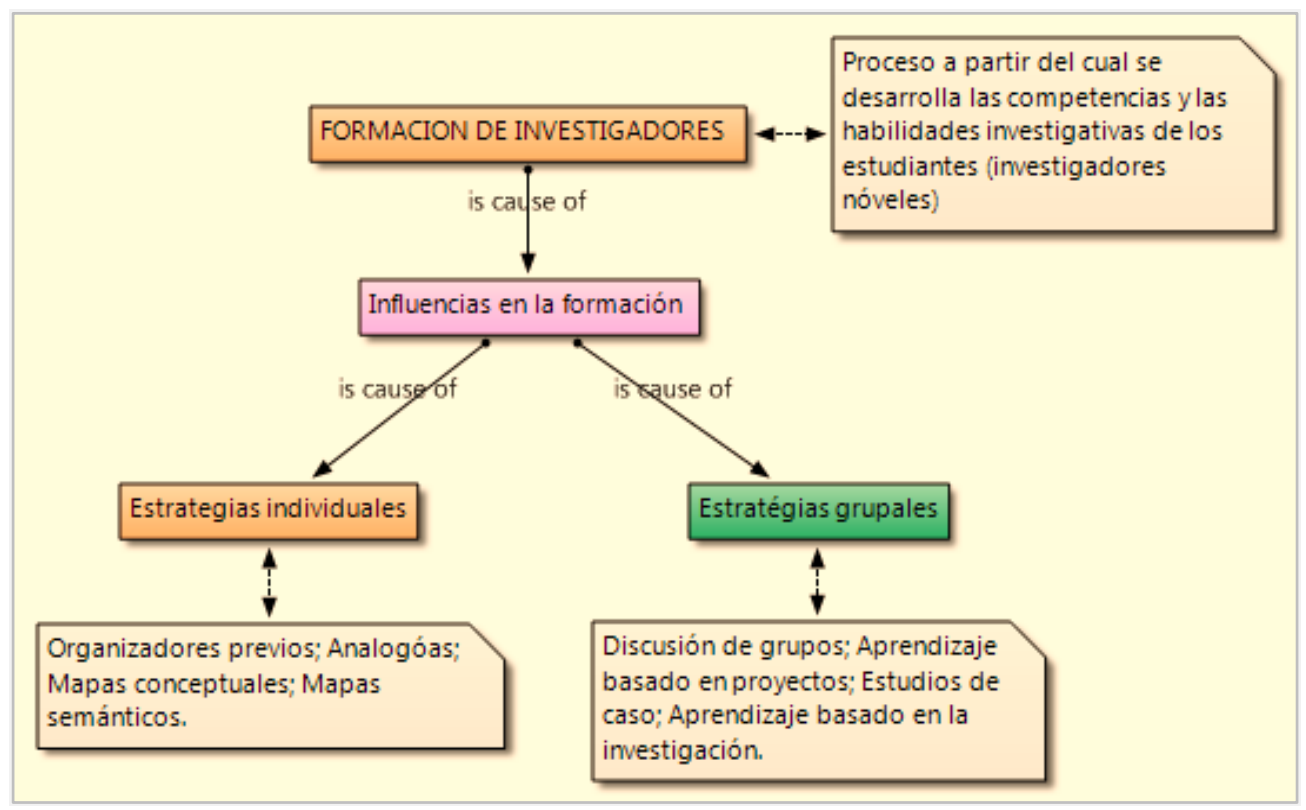

Fuente: Elaboración propia

La figura 1 expresa que los procesos de formación de investigadores se realizan a partir de un conjunto de estrategias; estas estrategias, se pueden dividir en dos grupos las estrategias individuales y las estrategias grupales. Las individuales están dirigidos a fortalecer las habilidades individuales y las de grupo, dirigido a fortalecer el análisis crítico, la discusión de grupos y la construcción de habilidades investigativas a nivel de grupo.

\section{Estrategias de investigación individual y grupal en tiempos de pandemia y distanciamiento social}

Una estrategia describe de manera clara el procedimiento de cómo se abordará el proceso de una investigación, principalmente la recolección de la información de campo, en base a un problema de estudio. En este sentido, y en base a los ejes de análisis y unidades de estudio del presente trabajo, se presentan los resultados de las entrevistas a expertos respecto a las estrategias de investigación en tiempos de pandemia y distanciamiento social; sin duda, los 
resultados evidencian que las estrategias investigativas posibles a ser utilizadas en las investigaciones sociales en tiempos de pandemia y distanciamiento social; a partir, de la educación disruptiva.

Figura 2

Estrategias de investigación en tiempos de pandemia y distanciamiento social

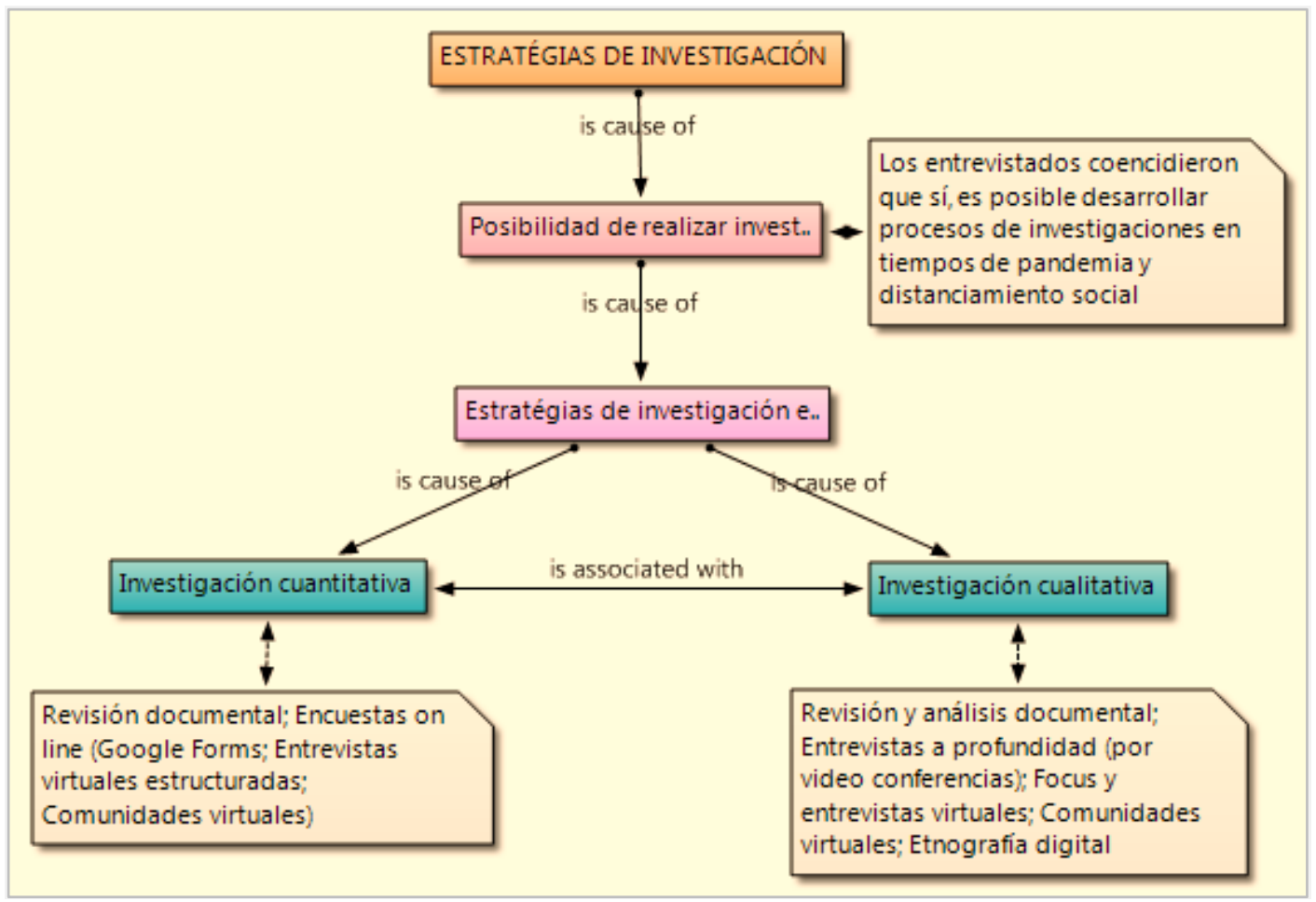

Fuente: Elaboración propia

La figura 2 pone en evidencia la posibilidad de realizar investigaciones sociales en tiempos de pandemia y distanciamiento social. Pese a las diferentes medidas impuestas por el estado respecto a la pandemia, con la finalidad de reducir índices de contagio. Los especialistas entrevistados en el presente estudio plantean la necesidad y la posibilidad de realizar procesos de investigación utilizando diferentes estrategias, basadas en las nuevas tecnologías de información y de comunicación (NTIC). Entre las estrategias más sobresalientes en la opinión de los especialistas - y en el contexto de la pandemia y el distanciamiento social — se puede mencionar a la revisión y análisis documental, las encuestas en línea, las entrevistas a profundidad utilizando video conferencias (como Zoom, Google Meet y otras plataformas), las 
entrevistas virtuales, las comunidades virtuales, la etnografía digital, etc. Que, pese a las medidas de distanciamiento social, permiten recolectar información relacionada a un tema de investigación.

\section{Discusión}

En respuesta al objetivo de esta investigación se destaca que, a partir de la educación disruptiva, existen nuevos desafíos en la formación de investigadores y el manejo de nuevas estrategias de indagación en tiempos de pandemia y distanciamiento social. No obstante, se toma en cuenta determinadas limitaciones y restricciones en el desarrollo de actividades educativas en el contexto de la educación superior basadas en las actividades tradicionales, como desarrollar clases presenciales, las prácticas institucionales y las investigaciones de campo (Carolan et al., 2020; Harris \& Jones, 2020; McMaster et al., 2020). Estas consideraciones hacen que la educación disruptiva, en el ambiente de educación superior, llamen interés a partir de los planes de contingencia que se han implementado en las universidades, la accesibilidad a nuevas herramientas y aprender a usarlas se convierte en otro desafío compartido que, de a pocos se van superando (Cabrol \& Severin, 2010; García-Morales et al., 2021).

En la línea de los expertos entrevistados, se confirma que la educación disruptiva trata de constituirse en una categoría que genera conocimientos y que coadyuva a generar formas de transmitir ese conocimiento, de acuerdo a los estudios de Sánchez-Alcaraz et al. (2021) y Suyambu et al. (2020). Lo anterior permite concluir la existencia en la actualidad de una corriente o forma de entender a la educación a partir de una interrupción de las prácticas y formas tradicionales de realizar la práctica educativa y los procesos de aprendizaje-enseñanza, una nueva forma de encarar los procesos educativos e investigativos; todo ello, a partir del uso de la tecnología y las nuevas plataformas educativas (Reich, 2020; Sunita, 2020).

Por otro lado, respecto a la categoría de formación de investigadores en ciencias sociales, se destaca que esto implica enseñar a investigar, teniendo como referente el proceso mismo de la generación efectiva del conocimiento científico (Sánchez, 2014); si se quiere enseñar a investigar, un aspecto importante es cambiar la didáctica de la investigación, pasando de una enseñanza teórica, a una didáctica práctica.

El planteamiento anterior pone en evidencia la necesidad de utilizar un conjunto de estrategias en el proceso de formación de investigadores; es decir, la didáctica de la 
investigación y la formación de investigadores, deben estar basados en estrategias prácticas, que permitan promover la capacitación y el entrenamiento de procesos investigativos en el terreno o área de investigación; a partir de ello, existe la necesidad de incluir en la formación de investigadores estrategias que permitan promover la práctica, el análisis y el pensamiento crítico, en función a situaciones reales. Es así, que un formador de investigadores debe ser un investigador, debe ser capaz de entender cómo se genera el conocimiento científico, pero sobre todo debe saber utilizar el mejor método para llegar a sus estudiantes (Carretero, 2019).

Lo anterior implica relucir la necesidad de una formación de investigadores, basado en la interrelación con el entorno sociocultural de un determinado país; de modo, que la formación de investigadores se constituya en una actividad basada en la práctica. Del mismo modo, en relación a las estrategias de investigación utilizadas en los tiempos de pandemia y distanciamiento social, se ha diversificado en función de dar respuestas a las nuevas formas de realizar procesos de investigación, basados a los nuevos contextos de distanciamiento social; de acuerdo con el planteamiento de Casimiro et al. (2020), las estrategias investigativas son los procesos que se utiliza para la motivación a los estudiantes a explorar, observar y preguntar; es decir, las estrategias de formación de investigadores se tratan de un conjunto de procedimientos que contribuyen a la recolección de información de una manera activa. Ahora bien, este proceso de investigación y generación de un nuevo conocimiento producto de la investigación científica, se ha visto abruptamente interrumpida producto de la pandemia COVID-19 y el distanciamiento social; a partir de ello, se ha iniciado nuevas formas de realizar procesos de investigación, utilizando nuevas estrategias como se ha podido evidenciar en la presente investigación.

Entre las limitaciones de esta investigación se destaca la manera de seleccionar a los informantes mediante el grado académico que poseen (maestro y doctor). Esa forma de seleccionar podría tener sesgos, ya que las habilidades de la percepción de los fenómenos sociales no se miden por los grados, sino por el desempeño y la experiencia vivida por los actores sociales. En ese sentido, se recomienda para las futuras investigaciones centrarse en las entrevistas con mayor cantidad de profesionales que hayan tenido experiencia empírica durante el proceso de enseñanza-aprendizaje con el contexto de la pandemia. 


\section{Conclusiones}

A partir de los nuevos hallazgos en el contexto de la educación disruptiva, esta constituye un referente importante en el planteamiento de nuevos desafíos en la formación de investigadores sociales, en el contexto de la pandemia y distanciamiento social. Este proceso permite identificar nuevas estrategias posibles a utilizar en la labor investigativa en épocas de emergencia sanitaria. En la categoría de formación de investigadores, es importante mencionar que, pese a la pandemia, la emergencia sanitaria y el distanciamiento social, los procesos de formación de investigadores nóveles en el nivel de grado (licenciatura) es una tarea fundamental que debe cumplir la universidad, en función de mejorar y fortalecer las competencias investigativas de los estudiantes.

En relación a la categoría de estrategias de investigación en tiempos de pandemia y distanciamiento social, la mayoría de los expertos/especialistas entrevistados plantean la posibilidad y la necesidad de realizar investigaciones sociales en tiempos de la covid19; $\sin$ embargo, frente a esta crisis sanitaria, es muy importante promover nuevas estrategias de investigación en este contexto que permitan la participación activa de los involucrados en las investigaciones. Por ello, y en base a los hallazgos y evidencias de la presente investigación, se sugiere profundizar y desarrollar otras investigaciones en torno a la educación disruptiva, su relación con la actual coyuntura y la elaboración de nuevas formas y estrategias para realizar investigaciones sociales en la actualidad.

\section{Referencias}

Abdel-Basset, M., Chang, V., \& Nabeeh, N. A. (2021). An intelligent framework using disruptive technologies for COVID-19 analysis. Technological Forecasting and Social Change, 163, 120431. https://doi.org/10.1016/j.techfore.2020.120431

Alvarez, J., Labraña, J., \& Brunner, J. J. (2021). La educación superior técnico profesional frente a nuevos desafíos: La Cuarta Revolución Industrial y la Pandemia por COVID-19. Revista Educación, Política y Sociedad, 6 (1), 11-38. https://doi.org/10.15366/reps2021.6.1.001

Ander-Egg, E. (2011). Aprender a investigar. Nociones básicas para la investigación social. Argentina: Editorial Brujas.

Beaud, S. (2018). El uso de la entrevista en las ciencias sociales. En defensa de la entrevista etnográfica. Revista Colombiana de Antropología, 54 (1), 175-218. https://doi.org/10.22380/2539472X.388 
Bonilla-Guachamín, J. A. (2020). Las dos caras de la educación en el COVID-19. CienciAmérica, 9 (2), 89. https://doi.org/10.33210/ca.v9i2.294

Cabrol, M., \& Severin, E. (2010). TICS en Educación: Una Innovación Disruptiva. https://publications.iadb.org/es/publicacion/14676/tics-en-educacion-una-innovaciondisruptiva

Camacho, Ó. (2010). La integración de la fe en el proceso de enseñanza-aprendizaje en el currículo formal de las universidades adventistas de México [Universidad de Montemorelos]. http://dspace.biblioteca.um.edu.mx/xmlui/handle/20.500.11972/834

Carolan, C., Davies, C. L., Crookes, P., McGhee, S., \& Roxburgh, M. (2020). COVID 19: Disruptive impacts and transformative opportunities in undergraduate nurse education. Nurse education in practice, 46, 102807. https://doi.org/10.1016/j.nepr.2020.102807

Carretero, S. (2019). Didáctica para la formación de investigadores desde la investigación vivencialista - intuitiva. Delectus, Revista científica, 2, 1-16. https://doi.org/https://doi.org/10.36996/delectus.v2i2.26

Casimiro, W., Casimiro, C., Ramos, F., \& Casimiro, J. (2020). Estrategias didácticas utilizadas por los docentes y actitudes investigativas de los estudiantes. Revista Conrado, 16, 175183. https://conrado.ucf.edu.cu/index.php/conrado/article/view/1469

Cepeda, J. (2014). Estrategias de enseñanza para el aprendizaje por competencias. México: UNID, Editorial Digital.

De Giusti, A. (2021). Reflexiones sobre Educación y Tecnología Post-Pandemia. Revista Iberoamericana de Tecnología en Educación y Educación en Tecnología, 28, e1. https://doi.org/10.24215/18509959.28.e1

Dibley, L., Norton, C., \& Whitehead, E. (2018). The experience of stigma in inflammatory bowel disease: An interpretive (hermeneutic) phenomenological study. Journal of Advanced Nursing, 74 (4), 838-851. https://doi.org/10.1111/jan.13492

Elgueta, M. F. (2020). Desafíos de la educación jurídica latinoamericana en tiempos de pandemia. Revista Pedagogía Universitaria y Didáctica del Derecho, 7 (1), 1. https://doi.org/10.5354/0719-5885.2020.57763

Fernández, M. (2002). La formación de investigadores cientificos en España. Centro de Investigaciones Sociológicas.

García-Morales, V. J., Garrido-Moreno, A., \& Martín-Rojas, R. (2021). The Transformation of Higher Education After the COVID Disruption: Emerging Challenges in an Online $\begin{array}{lllll}\text { Learning } & \text { Scenario. } & \text { Frontiers }\end{array}$ https://doi.org/10.3389/fpsyg.2021.616059

Gonzales, J., Galindo, N., Galindo, J., \& Gold, M. (2004). Los paradigmas de la calidad educativa. De la autoevaluación a la acreditación (G. Rodrigues, O. Gonzalez, \& D. Bento 
(eds.); Primera ed). Editorial UDUAL.

González-Revaldería, J., Holguín-Holgado, P., Lumbreras-Marín, E., \& Núñez-López, G. (2017). La entrevista en profundidad y la metodología Kano para conocer los requisitos de los usuarios en una unidad de quemados. Revista de Calidad Asistencial, 32 (1), 21-26. https://doi.org/10.1016/j.cali.2016.06.009

Harris, A., \& Jones, M. (2020). COVID 19 - school leadership in disruptive times. School $\begin{array}{lllll}\text { Leadership } \quad \text { \& } & \text { Management, } & 40 \quad \text { (4), }\end{array}$ https://doi.org/10.1080/13632434.2020.1811479

Hernández, R., Fernández, C., \& Baptista, P. (2014). Metodología de la Investigación (6ta ed.). McGRAW-HILL / Interamericana Editores.

Ho, K. H. M., Chiang, V. C. L., \& Leung, D. (2017). Hermeneutic phenomenological analysis: the 'possibility' beyond 'actuality' in thematic analysis. Journal of Advanced Nursing, 73 (7), 1757-1766. https://doi.org/10.1111/jan.13255

Iivari, N. (2018). Using member checking in interpretive research practice. Information Technology \& People, 31 (1), 111-133. https://doi.org/10.1108/ITP-07-2016-0168

Landazábal, D. P., Páez, D. I., \& Pineda, E. (2013). Diseño de una innovación pedagógica para la formación en investigación apoyada en ambientes digitales. Revista Virtual Universidad Católica del Norte, 40, 4-30. https://www.redalyc.org/articulo.oa?id=194229200002

McMaster, D., Veremu, M., \& Santucci, C. (2020). COVID-19: opportunities for professional development and disruptive innovation. The clinical teacher, 17 (3), 238-240. https://doi.org/10.1111/tct.13175

Millán Núñez-Cortés, J. (2020). COVID-19 por SARS-Cov2 también ha afectado a la Educación Médica. Educación Médica, $21 \quad$ (4), 261-264. https://doi.org/10.1016/j.edumed.2020.06.001

Molano, M. (2018). Educación disruptiva en el contexto lasallista: glosas para la discusión. Revista Universidad de La Salle, 1 (75), 55-68. https://doi.org/10.19052/ruls.vol1.iss75.4

Ordorika, I. (2020). Pandemia y educación superior. Revista de la educación superior, 49 (194), 1-8. https://doi.org/https://doi.org/10.36857/resu.2020.194.1120

Pimienta-Prieto, J. (2012). Estrategias de enseñanza-aprendizaje (1ra. Ed.). Pearson Educación de México, S.A.

Piyatamrong, T., Derrick, J., \& Nyamapfene, A. (2021). Technology-Mediated Higher Education Provision during the COVID-19 Pandemic: A Qualitative Assessment of Engineering Student Experiences and Sentiments. Journal of Engineering Education Transformations, 34, 290. https://doi.org/10.16920/jeet/2021/v34i0/157158

Reich, J. (2020). Introduction: Education Technology's Unrequited Disruption. En Failure to Disrupt (pp. 1-14). Harvard University Press. https://doi.org/10.4159/9780674249684-002 
Rivas, L. (2004). La formación de investigadores en México. Revista Perfiles Latinoamericanos, 12

(25),

89-113. https://perfilesla.flacso.edu.mx/index.php/perfilesla/article/view/250

Sánchez-Alcaraz, B. J., Gómez-Mármol, A., Valero-Valenzuela, A., \& Courel-Ibáñez, J. (2021). Implementation of the Teaching Personal and Social Responsibility Model to Reduce Violent and Disruptive Behaviors in Adolescents Through Physical Activity: A Quantitative Approach. Journal of Teaching in Physical Education, 40 (2), 238-244. https://doi.org/10.1123/jtpe.2019-0126

Sánchez, R. (2014). Enseñar a investigar. Una didáctica nueva de la investigación en ciencias sociales y humanas (Segunda ed). Plaza y Valdés S.A.

Sordini, M. V. (2018). La entrevista en profundidad en el ámbito de la gestión pública. Revista Reflexiones, 98 (1), 75-88. https://doi.org/10.15517/rr.v98i1.33083

Sunita, M.-L. (2020). Education in the Era of COVID-19: Innovative Solutions to Real Challenges. The Educational Review, 4 (11), 193-198. https://doi.org/10.26855/er.2020.11.002

Suyambu, G. T., Anand, M., \& Janakirani, M. (2020). Blockchain - A Most Disruptive Technology On The Spotlight Of World Engineering Education Paradigm. Procedia Computer Science, 172, 152-158. https://doi.org/10.1016/j.procs.2020.05.023

Vélez, D., \& Pérez, R. (2019). Filosofía y didáctica en la formación de investigadores México: Editorial Laripse. 